PROCEEDINGS OF THE

AMERICAN MATHEMATICAL SOCIETY

Volume 132, Number 6, Pages 1765-1773

S 0002-9939(03)07278-2

Article electronically published on October 24, 2003

\title{
A BANACH SPACE WITH THE SCHUR AND THE DAUGAVET PROPERTY
}

\author{
VLADIMIR KADETS AND DIRK WERNER
}

(Communicated by N. Tomczak-Jaegermann)

\begin{abstract}
We show that a minor refinement of the Bourgain-Rosenthal construction of a Banach space without the Radon-Nikodým property which contains no bounded $\delta$-trees yields a space with the Daugavet property and the Schur property. Using this example we answer some open questions on the structure of such spaces; in particular, we show that the Daugavet property is not inherited by ultraproducts.
\end{abstract}

\section{INTRODUCTION}

A Banach space $X$ is said to have the Daugavet property if every rank-1 operator $T: X \rightarrow X$ satisfies

$$
\|\mathrm{Id}+T\|=1+\|T\| .
$$

Examples include $C[0,1], L_{1}[0,1]$, certain function algebras such as the disk algebra or $H^{\infty}$ and some of their subspaces and quotients. Such spaces are studied in detail in 6 .

It is known that a space with the Daugavet property cannot have the RadonNikodým property [11, that a space with the Daugavet property contains a copy of $\ell_{1}$ [6], and that in a space with the Daugavet property not only rank-1 operators, but also weakly compact operators, strong Radon-Nikodým operators, operators not fixing a copy of $\ell_{1}$ and their linear combinations satisfy the Daugavet equation (1.1); see [6], 7], 9]. A space with the Daugavet property does not have an unconditional basis [4] and does not even embed into a space with an unconditional basis [6].

A Banach space has the Schur property if every weakly convergent sequence converges in norm. It has been asked in 5 and 10 whether there exists a Banach space that has both the Schur and the Daugavet property. Given the isomorphic properties that the Daugavet property entails as listed above, the two properties might appear to be mutually exclusive since a space with the Daugavet property should be thought of as rather large whereas a Schur space, which is hereditarily $\ell_{1}$, could be imagined as rather thin. In the other direction, previous studies of the Daugavet property [7] have supported the conjecture that a space with the

Received by the editors February 13, 2003.

2000 Mathematics Subject Classification. Primary 46B04; Secondary 46B20, 46M07.

Key words and phrases. Daugavet property, Schur property, ultraproducts of Banach spaces.

The work of the first author was supported by a fellowship from the Alexander-von-Humboldt Stiftung.

(C)2003 American Mathematical Society 
Daugavet property always contains a copy of $\ell_{2}$. (This conjecture is clearly not compatible with the Schur space conjecture.)

In this paper we point out that a certain subspace of $L_{1}$ that was constructed by Bourgain and Rosenthal [3] indeed has the Schur and the Daugavet property. This is shown in Section 2 where we review the Bourgain-Rosenthal construction along with some minor modifications to achieve our result.

In 2 we studied the Daugavet property for ultraproducts and asked whether it is stable under ultraproducts. In Section 3 we show that direct sums of certain Bourgain-Rosenthal spaces serve as counterexamples. To explain why the BourgainRosenthal spaces might be relevant to this topic we need some technical notions; so we resume this discussion only in Section 3.

Throughout the paper we will need the following geometrical characterization of the Daugavet property from [6] Lemma 2.2]. We denote by $B(X)$ the closed unit ball of $X$ and by $S(X)$ its unit sphere, and we recall that a slice of the unit ball is a nonvoid set of the form $S=\left\{x \in B(X)\right.$ : $\left.\operatorname{Re} x^{*}(x)>\alpha\right\}$ for some functional $x^{*} \in X^{*}$.

Lemma 1.1. The following assertions are equivalent:

(i) $X$ has the Daugavet property.

(ii) For every $x \in S(X), \varepsilon>0$ and every slice $S$ of $B(X)$ there exists some $y \in S$ such that $\|x+y\| \geq 2-\varepsilon$.

For an element $x \in S(X)$ and $\varepsilon>0$ denote

$$
l^{+}(x, \varepsilon)=\{y \in S(X):\|x+y\| \geq 2-\varepsilon\} .
$$

It is immediate from Lemma 1.1 and the Hahn-Banach theorem that the Daugavet property can be characterized by means of the sets $l^{+}(x, \varepsilon)$ as follows.

Lemma 1.2. The following assertions are equivalent:

(i) $X$ has the Daugavet property.

(ii) For every $x \in S(X)$ and $\varepsilon>0$ the set $\operatorname{conv}\left(l^{+}(x, \varepsilon)\right)$, the convex hull of $l^{+}(x, \varepsilon)$, is dense in $B(X)$.

\section{The Bourgain-Rosenthal Example Revisited}

In this section we will present an example of a Banach space that has both the Schur and the Daugavet property. It belongs to the class of spaces that Bourgain and Rosenthal have constructed in [3]. However, we base our exposition on the one that Benyamini and Lindenstrauss have given in [1] and not on the original paper.

Below we consider the space $L_{1}=L_{1}(\Omega, \Sigma, \mu)$ over a separable nonatomic measure space. The symbol $\|$. $\|$ will refer to the $L_{1}$-norm. Besides the norm topology we will also consider the topology of convergence in measure, generated by the metric

$$
d(f, g)=\inf \{\varepsilon>0: \mu\{t:|f(t)-g(t)| \geq \varepsilon\} \leq \varepsilon\} .
$$

We first observe a simple lemma on $L_{1}$-orthogonality that will be used later. In particular, it applies to bounded subsets of finite-dimensional, indeed reflexive, subspaces of $L_{1}$.

Lemma 2.1. Let $H$ be a uniformly integrable subset of $L_{1}$ and $\varepsilon>0$. Then there is a $\delta>0$ such that for every $g \in H$ and every $f \in L_{1}$ with $d(f, 0)<\delta$ the following inequality holds:

$$
\|f+g\| \geq\|f\|+\|g\|-\varepsilon
$$


Proof. Using the uniform integrability of $H$ one can find a $\delta>0$ such that

$$
2 \int_{A}|g| d \mu+2 \delta<\varepsilon
$$

for every $g \in H$ and every measurable subset $A \subset \Omega$ with $\mu(A)<\delta$. Now fix $f \in L_{1}$ with $d(f, 0)<\delta$ and denote $A=\{t:|f(t)| \geq \delta\}$. Then for every $g \in H$ we have

$$
\begin{aligned}
\|f+g\| & =\int_{A}|f+g| d \mu+\int_{\Omega \backslash A}|f+g| d \mu \\
& \geq \int_{A}|f| d \mu+\int_{\Omega \backslash A}|g| d \mu-\int_{A}|g| d \mu-\int_{\Omega \backslash A}|f| d \mu \\
& \geq\|f\|+\|g\|-2 \int_{A}|g| d \mu-2 \delta \\
& \geq\|f\|+\|g\|-\varepsilon
\end{aligned}
$$

as requested.

We now quote Lemma 5.26 from [1] that contains the key to the BourgainRosenthal construction.

Lemma 2.2. Let $0<\varepsilon<1$. Then there is a function $f \in L_{1}[0,1]$ with the following properties:

(a) $f \geq 0,\|f\|=1$ and $\|f-\mathbf{1}\| \geq 2-\varepsilon$.

(b) Let $\left\{f_{j}\right\}_{j=1}^{\infty} \subset L_{1}[0,1]$ be a sequence of independent random variables with the same distribution as $f$. Then for every $g \in \varlimsup \operatorname{lin}\left\{f_{j}\right\}_{j=1}^{\infty}$ with $\|g\| \leq 1$, there is a constant function $c$ with $d(g, c) \leq \varepsilon$.

(c) $\left\|n^{-1} \sum_{j=1}^{n} f_{j}-\mathbf{1}\right\| \rightarrow 0$ as $n \rightarrow \infty$.

Remark 2.3. Property (a) of the previous lemma implies, in particular, that $d(f, 0) \leq$ $\sqrt{\varepsilon}$. Indeed, for $A=\{t: f(t) \geq \sqrt{\varepsilon}\}$ we have ( $\lambda$ denotes the Lebesgue measure)

$$
\int_{A}|f(t)-1| d t \leq \int_{A}(f(t)+1) d t-\sqrt{\varepsilon} \lambda(A)
$$

since $(a+1)-|a-1|=2 \min \{a, 1\} \geq \sqrt{\varepsilon}$ for $a \geq \sqrt{\varepsilon}$; so

$$
2-\varepsilon \leq\|f-\mathbf{1}\| \leq 2-\sqrt{\varepsilon} \lambda(A)
$$

and hence $\lambda(A) \leq \sqrt{\varepsilon}$.

In the lemma and in the construction below, $(\Omega, \Sigma, \mu)$ will be the product of countably many copies of the probability space $[0,1]$. A subspace of $L_{1}=$ $L_{1}(\Omega, \Sigma, \mu)$ is said to depend on finitely many coordinates if all the elements of the subspace are functions depending only on a common finite set of coordinates.

The next lemma is a refinement of [1. Lemma 5.27]; the difference is that the latter lemma claims (a) only for $u=u_{k}$.

Lemma 2.4. Let $G$ be a finite-dimensional subspace of $L_{1}$ that depends on finitely many coordinates. Let $\left\{u_{k}\right\}_{k=1}^{m} \subset S(G)$ and $\varepsilon>0$. Then there is a finitedimensional subspace $F \subset L_{1}$, also depending on finitely many coordinates and containing $G$, and there are an integer $n$ and functions $\left\{v_{k, j}\right\}_{k \leq m, j \leq n} \subset S(F)$ such that:

(a) $\left\|u+v_{k, j}\right\| \geq 2-\varepsilon$ for every $u \in S(G)$ and all $k$ and $j$;

(b) $\left\|u_{k}-n^{-1} \sum_{j=1}^{n} v_{k, j}\right\| \leq \varepsilon$ for all $k$; 
(c) for every $\varphi \in B(F)$ there is a $\psi \in B(G)$ with $d(\varphi, \psi) \leq \varepsilon$.

Proof. We shall first recall the construction of the proof in [1, Lemma 5.27], and then point out the necessary changes for our proof.

Let $\varepsilon_{1}>0$ be small enough, let $f$ be the function given by Lemma 2.2 for this $\varepsilon_{1}$, and let $n$ be such that $\left\|n^{-1} \sum_{j=1}^{n} f_{j}-\mathbf{1}\right\| \leq \varepsilon_{1}$ for $f_{j}$ as in Lemma 2.2. (It will become apparent at the end of the proof how small $\varepsilon_{1}$ should be chosen.) Let $G$ depend on the first $N$ coordinates of $\Omega$. For every $k \leq m$, choose $\left\{f_{k, j}\right\}_{j \leq n}$ that depend on the $(N+k)$-th coordinate of $\Omega$ and are equidistributed with $\left\{f_{j}\right\}_{j \leq n}$. Put $v_{k, j}=f_{k, j} u_{k}$, and let $F$ be the span of $G$ and of $\left\{v_{k, j}\right\}_{k \leq m, j \leq n}$. Note that $\left\|f_{k, j} u_{k}\right\|=1$ since the two functions are stochastically independent.

The properties (b) and (c) are the same as in Lemma 5.27 from [1] (cf. 1, p. 118] for the norm-1 part of (c)). So we need not repeat their proofs here. We only have to deal with property (a). According to Remark [2.3, $d\left(f_{k, j}, 0\right) \leq \sqrt{\varepsilon_{1}}$. Denote

$$
A=A_{k, j}=\left\{t: f_{k, j}(t) \geq \sqrt{\varepsilon_{1}}\right\}, \quad v_{k, j}^{1}=f_{k, j} u_{k} \chi_{A}, \quad v_{k, j}^{2}=f_{k, j} u_{k} \chi_{\Omega \backslash A} .
$$

Then

$$
v_{k, j}=v_{k, j}^{1}+v_{k, j}^{2},
$$

where the first summand has a small support, namely

$$
\mu\left(\operatorname{supp} v_{k, j}^{1}\right) \leq \mu(A) \leq \sqrt{\varepsilon_{1}},
$$

and the second summand has a small norm, namely

$$
\left\|v_{k, j}^{2}\right\| \leq \int_{\Omega \backslash A} \sqrt{\varepsilon_{1}}\left|u_{k}\right| d \mu \leq \sqrt{\varepsilon_{1}} .
$$

So for every $u \in S(G)$,

$$
\left\|u+v_{k, j}\right\| \geq\left\|u+v_{k, j}^{1}\right\|-\sqrt{\varepsilon_{1}},
$$

and $d\left(v_{k, j}^{1}, 0\right) \leq \sqrt{\varepsilon_{1}}$. To finish the proof it is enough to apply Lemma 2.1

We now turn to the actual construction of the example. Fix a decreasing sequence of numbers $\varepsilon_{N}>0$ with $\sum_{j=N+1}^{\infty} \varepsilon_{j}<\varepsilon_{N}$ for all $N \in \mathbb{N}$ and select inductively finite-dimensional subspaces of $L_{1}$,

$$
\operatorname{lin}\{\mathbf{1}\}=E_{1} \subset E_{2} \subset E_{3} \subset \ldots,
$$

each of them depending on finitely many coordinates, $\varepsilon_{N}$-nets $\left\{u_{k}^{N}\right\}_{k=1}^{m(N)}$ of $S\left(E_{N}\right)$ and collections of elements $\left\{v_{k, j}^{N}\right\}_{k \leq m(N), j \leq n(N)} \subset S\left(E_{N+1}\right)$ in such a way that the conclusion of Lemma 2.4 holds with $\varepsilon=\varepsilon_{N}, G=E_{N}, F=E_{N+1},\left\{u_{k}\right\}_{k=1}^{m}=$ $\left\{u_{k}^{N}\right\}_{k=1}^{m(N)},\left\{v_{k, j}\right\}_{k \leq m, j \leq n}=\left\{v_{k, j}^{N}\right\}_{k \leq m(N), j \leq n(N)}$. Denote $E=\overline{\bigcup_{N=1}^{\infty} E_{N}}$.

Only part (a) in the next theorem is new; the other parts are due to Bourgain and Rosenthal, but we sketch the proofs for completeness.

Theorem 2.5. The space $E$ constructed above has the following properties:

(a) E has the Daugavet property;

(b) for every $f \in B(E)$ and for every $N \in \mathbb{N}$ there exists a $g \in B\left(E_{N}\right)$ such that $d(f, g)<\varepsilon_{N}$;

(c) E has the Schur property. 
Proof. (a) According to Lemma 1.2 we need to show that for every $u \in S(E)$ and $\varepsilon>0$ the set $\operatorname{conv}\left(l^{+}(u, \varepsilon)\right)$ is dense in $B(E)$. By a perturbation argument it is enough to check this condition only for $u$ from the dense subset $S\left(\bigcup_{N=1}^{\infty} E_{N}\right)$ of $S(E)$.

Fix an $N \in \mathbb{N}, u \in S\left(E_{N}\right)$ and $\varepsilon>0$. There is an $M>N$ such that $\varepsilon_{M}<\varepsilon$. By construction (property (a) of Lemma 2.4) all the elements $v_{k, j}^{L}$ with $L>M$ belong to $l^{+}(u, \varepsilon)$. Taking into account property (b) of Lemma 2.4 and the fact that $\left\{u_{k}^{L}\right\}_{k=1}^{m(L)}$ forms an $\varepsilon_{L}$-net of $S\left(E_{L}\right)$, one can easily establish the density of $\operatorname{conv}\left(l^{+}(u, \varepsilon)\right)$ in $B(E)$.

(b) Fix an $f \in B\left(\bigcup_{J=1}^{\infty} E_{J}\right)$ and an $N \in \mathbb{N}$. Then $f \in B\left(E_{N+L}\right)$ for some $L$. Applying property (c) of Lemma 2.4 to $f$ we find an $f_{1} \in S\left(E_{N+L-1}\right)$ with $d\left(f, f_{1}\right) \leq \varepsilon_{N+L}$. Applying again property (c) of Lemma 2.4 to $f_{1}$ we find an $f_{2} \in S\left(E_{N+L-2}\right)$ with $d\left(f_{1}, f_{2}\right) \leq \varepsilon_{N+L-1}$. Continuing in this fashion we obtain in the $L$-th step some $g=f_{L} \in S\left(E_{N}\right)$ for which

$$
d(f, g) \leq \varepsilon_{N+L}+\varepsilon_{N+L-1}+\cdots+\varepsilon_{N+1}<\varepsilon_{N} .
$$

(c) It follows from (b) that the unit ball of $E$ is a precompact in the metric $d$ of convergence in measure. This implies the Schur property. (In fact, a stronger conclusion can be drawn, namely, that $E$ has the strong Schur property; see [3].)

We observe that the space $E$ cannot be a rich subspace of $L_{1}$ in the terminology of [7. Indeed, the unit ball of $E$ is precompact in the metric of convergence in measure; hence its $\tau$-closure $C_{E}$ is compact for that topology $\tau$. But if $E$ were rich, by [5, Prop. 2.2], $C_{E}$ would contain $\frac{1}{2} B\left(L_{1}\right)$, and $B\left(L_{1}\right)$ would be $\tau$-compact as well, which is clearly false. This remark reveals that $E$ is an essentially new specimen among the spaces with the Daugavet property.

Since a Banach space with the Schur property cannot contain infinite-dimensional reflexive subspaces, the following corollary holds. It answers Question (4) from [7].

Corollary 2.6. There exists a Banach space with the Daugavet property that fails to contain infinite-dimensional reflexive subspaces; in particular, it fails to contain a copy of $\ell_{2}$.

It follows as well that the space $E$ from Theorem 2.5 does not contain a copy of $L_{1}$; the first space with the Daugavet property having this feature was constructed in [6] after an example given by Talagrand.

One can likewise express Corollary [2.6] in terms of narrow operators, a notion studied in detail in [7]. Namely, the identity operator on $E$ is an operator that does not fix a copy of $\ell_{2}$, yet it is not narrow. This remark provides a negative answer to Question (5) from [7].

Schmidt [8] proved that every Dunford-Pettis operator $T$ on $L_{1}$ (i.e., $T$ maps weakly convergent sequences to norm convergent sequences) satisfies the Daugavet equation (1.1). In fact, such an operator is easily seen to be narrow on $L_{1}$. However, on the Schur space $E$ above, -Id is Dunford-Pettis, but clearly fails (1.I). Therefore, we have:

Corollary 2.7. There is a Banach space with the Daugavet property and a DunfordPettis operator on that space that fails (1.1). Hence, in general, Dunford-Pettis operators are not narrow. 


\section{The Uniform DAugaVet PROPERTY AND Ultraproducts}

In Section 6 of [2], conditions for an ultraproduct of Banach spaces to possess the Daugavet property were discussed. For the reader's convenience we recall the relevant notions and results.

For a subset $A \subset X$ we denote by $\operatorname{conv}_{n}(A)$ the set of all convex combinations of all $n$-point collections of elements from $A$. Clearly, $\operatorname{conv}(A)=\bigcup_{n \in \mathbb{N}} \operatorname{conv}_{n}(A)$. Denote

$$
\operatorname{Daug}_{n}(X, \varepsilon)=\sup _{x, y \in S(X)} \operatorname{dist}\left(\operatorname{conv}_{n}\left(l^{+}(x, \varepsilon)\right), y\right)
$$

where $\operatorname{dist}(A, B)$ denotes the distance between two subsets, i.e., $\operatorname{dist}(A, B)=$ $\inf \{\|a-b\|: a \in A, b \in B\}$.

It is easy to see that for every $\varepsilon>0$, the sequence $\left(\operatorname{Daug}_{n}(X, \varepsilon)\right)$ decreases.

Definition 3.1. A Banach space $X$ is said to have the uniform Daugavet property if for every $\varepsilon>0$, the sequence $\left(\operatorname{Daug}_{n}(X, \varepsilon)\right.$ ) tends to 0 when $n$ tends to infinity.

It follows from Lemma 1.2 that the uniform Daugavet property implies the Daugavet property.

We now recall a special case of [2, Th. 6.2].

Theorem 3.2. Let $\mathcal{U}$ be a free ultrafilter on $\mathbb{N},\left\{X_{i}\right\}_{i \in \mathbb{N}}$ a collection of Banach spaces and $X$ the corresponding ultraproduct of the $X_{i}$. Then the following assertions are equivalent:

(i) $X$ has the Daugavet property.

(ii) For every $\varepsilon>0, \lim _{\mathcal{U}, n} \operatorname{Daug}_{n}\left(X_{i}, \varepsilon\right)=0$. In other words, for every fixed $\varepsilon>0$ and every $\delta>0$ there is an $n \in \mathbb{N}$ such that the set of all $i$ for which $\operatorname{Daug}_{n}\left(X_{i}, \varepsilon\right) \leq \delta$ belongs to the ultrafilter $\mathcal{U}$.

In particular, a Banach space $Y$ has the uniform Daugavet property if and only if the ultrapower $Y^{\mathcal{U}}$ has the Daugavet property.

Proof. To deduce (i) from (ii) one just has to notice that if

$$
\left\{i \in \mathbb{N}: \operatorname{Daug}_{n}\left(X_{i}, \varepsilon\right) \leq \delta\right\} \in \mathcal{U},
$$

then $\operatorname{Daug}_{n}(X, \varepsilon) \leq \delta$. So (ii) implies that $\operatorname{Daug}_{n}(X, \varepsilon)$ tends to 0 when $n \rightarrow \infty$ for every $\varepsilon>0$, which proves the Daugavet property for $X$.

To deduce (ii) from (i) let us argue ad absurdum. Suppose there are $\varepsilon>0$ and $\delta>0$ such that for every $n \in \mathbb{N}$ the set $A_{n}=\left\{i \in \mathbb{N}: \operatorname{Daug}_{n}\left(X_{i}, \varepsilon\right)>\delta\right\}$ belongs to the ultrafilter $\mathcal{U}$. The sequence $\left(A_{n}\right)$ is descending, but its intersection need not be empty. However, deleting if necessary a finite number of elements in each of the $A_{n}$, we can find sets $B_{n} \in \mathcal{U}, B_{n} \subset A_{n}, B_{1} \supset B_{2} \supset \ldots$ such that $\bigcap_{n} B_{n}=\emptyset$.

If $i \in B_{n} \backslash B_{n+1}$, we can find $x_{i}, y_{i} \in S\left(X_{i}\right)$ such that

$$
\operatorname{dist}\left(\operatorname{conv}_{n}\left(l^{+}\left(x_{i}, \varepsilon\right)\right), y_{i}\right)>\delta \text {. }
$$

Hence, whenever $i \in B_{n}=\bigcup_{m=n}^{\infty} B_{m} \backslash B_{m+1}\left(\right.$ since $\left.\bigcap_{m} B_{m}=\emptyset\right)$, then

$$
\operatorname{dist}\left(\operatorname{conv}_{n}\left(l^{+}\left(x_{i}, \varepsilon\right)\right), y_{i}\right)>\delta \text {. }
$$

This implies for $x=\left(x_{i}\right) \in S(X), y=\left(y_{i}\right) \in S(X)$ that

$$
\operatorname{dist}\left(\operatorname{conv}_{n}\left(l^{+}(x, \varepsilon)\right), y\right) \geq \delta
$$

for every $n \in \mathbb{N}$ and thus

$$
\operatorname{dist}\left(\operatorname{conv}\left(l^{+}(x, \varepsilon)\right), y\right) \geq \delta
$$


contradicting the Daugavet property of $X$.

It was asked in 2] whether the Daugavet property and its uniform version are actually equivalent. That the Bourgain-Rosenthal spaces may shed some light on this question is suggested by the following facts. The Bourgain-Rosenthal spaces were constructed in order to provide an example of a Banach space that fails the Radon-Nikodým property (i.e., some uniformly bounded martingale diverges), yet every uniformly bounded dyadic martingale converges. Using more horticultural language, one can express this by saying that the unit ball contains some $\eta$-bush, but no $\eta$-trees (see Chapter 5 in 1 for these concepts) or indeed no $\eta$-bushes with a fixed number of branches at each branching node.

This is reminiscent of the Daugavet property and its uniform variant. The Daugavet property means that every $y$ of norm 1 is almost a convex combination of vectors from $l^{+}(x, \varepsilon)$ for any given $x$ of norm 1 ; this enables one to find an $\eta$-bush for any $\eta<2$. By contrast, the uniform Daugavet property is related to finding such bushes with a fixed number of branches at each level.

Precisely, we shall now prove the following theorem.

\section{Theorem 3.3.}

(a) For every $n \in \mathbb{N}$ there is a Banach space $X_{n}$ with the Daugavet property such that

$$
\operatorname{Daug}_{n}\left(X_{n}, \frac{1}{4}\right) \geq \frac{1}{2}
$$

(b) There is a Banach space $X$ which has the Daugavet property, but does not have the uniform Daugavet property.

(c) An ultrapower of a space with the Daugavet property does not necessarily possess the Daugavet property.

Proof. (a) We take as $X_{n}$ the space $E$ from Theorem 2.5 with parameters $\left\{\varepsilon_{j}\right\}_{j \in \mathbb{N}}$, where $\varepsilon_{1}$ is selected in such a way that for every constant function $g \in[-2,2]$ and every $f \in L_{1}$ with $d(f, 0)<n \varepsilon_{1}$ the inequality

$$
\|g+f\| \geq\|g\|+\|f\|-\frac{1}{4}
$$

holds (see Lemma 2.1). To prove (3.1) we check that

$$
\operatorname{dist}\left(\operatorname{conv}_{n}\left(l^{+}\left(x, \frac{1}{4}\right)\right), y\right) \geq \frac{1}{2}
$$

for the constant functions $x=-\mathbf{1}$ and $y=\mathbf{1}$.

Consider an arbitrary element $z \in \operatorname{conv}_{n}\left(l^{+}\left(x, \frac{1}{4}\right)\right), z=\sum_{k=1}^{n} \lambda_{k} z_{k}$, where $\lambda_{k} \geq$ $0, \sum_{k=1}^{n} \lambda_{k}=1,\left\|z_{k}\right\| \leq 1$ and

$$
\left\|z_{k}-\mathbf{1}\right\| \geq 7 / 4
$$

According to (b) of Theorem 2.5 (with $N=1$ and $f=z_{k}$ ), for every $k \leq n$ there is a constant function $\alpha_{k} \in[-1,1]$ such that $d\left(z_{k}-\alpha_{k}, 0\right)=d\left(z_{k}, \alpha_{k}\right)<\varepsilon_{1}$. Then, using (3.4) and (3.2) we conclude

$$
\begin{aligned}
1 \geq\left\|z_{k}\right\| & =\left\|\alpha_{k}+\left(z_{k}-\alpha_{k}\right)\right\| \\
& \geq\left|\alpha_{k}\right|+\left\|z_{k}-\alpha_{k}\right\|-\frac{1}{4} \\
& \geq\left|\alpha_{k}\right|+\left\|z_{k}-\mathbf{1}\right\|-\left|1-\alpha_{k}\right|-\frac{1}{4} \\
& \geq\left|\alpha_{k}\right|-\left|1-\alpha_{k}\right|+\frac{3}{2}
\end{aligned}
$$


therefore,

$$
\left|\alpha_{k}\right|-\left|1-\alpha_{k}\right| \leq-\frac{1}{2}
$$

This implies that $\alpha_{k} \leq 1 / 4$, and consequently

$$
\sum_{k=1}^{n} \lambda_{k} \alpha_{k} \leq 1 / 4
$$

Since $d\left(\sum_{k=1}^{n} \lambda_{k}\left(z_{k}-\alpha_{k}\right), 0\right)<n \varepsilon_{1}$, using (3.5) and (3.2) we deduce that

$$
\begin{aligned}
\|y-z\| & =\left\|\mathbf{1}-\sum_{k=1}^{n} \lambda_{k} z_{k}\right\| \\
& =\left\|\left(\mathbf{1}-\sum_{k=1}^{n} \lambda_{k} \alpha_{k}\right)+\sum_{k=1}^{n} \lambda_{k}\left(\alpha_{k}-z_{k}\right)\right\| \\
& \geq\left\|\mathbf{1}-\sum_{k=1}^{n} \lambda_{k} \alpha_{k}\right\|-\frac{1}{4} \geq \frac{1}{2},
\end{aligned}
$$

which proves (3.3).

(b) It is enough to take the $\ell_{1}$-direct sum $X=X_{1} \oplus_{1} X_{2} \oplus_{1} \ldots ; X$ has the Daugavet property by [11].

(c) This follows from (b) and Theorem 3.2 ,

\section{REFERENCES}

[1] Y. Benyamini and J. Lindenstrauss. Geometric Nonlinear Functional Analysis, Vol. 1. Colloquium Publications no. 48. Amer. Math. Soc., Providence, RI, 2000. MR 2001b:46001

[2] D. Bilik, V. M. Kadets, R. V. Shvidkoy And D. Werner. Narrow operators and the Daugavet property for ultraproducts. To appear in Positivity. Preprint available from http://xxx.lanl.gov.

[3] J. Bourgain and H. P. Rosenthal. Martingales valued in certain subspaces of $L^{1}$. Israel J. Math. 37 (1980), 54-75. MR 82g:46044

[4] V. M. KADETS. Some remarks concerning the Daugavet equation. Quaestiones Math. 19 (1996), 225-235. MR 97c:46015

[5] V. M. Kadets, N. Kalton, And D. Werner. Remarks on rich subspaces of Banach spaces. To appear in Studia Math. Preprint available from http://xxx.lanl.gov.

[6] V. M. Kadets, R. V. Shvidkoy, G. G. Sirotkin, and D. Werner. Banach spaces with the Daugavet property. Trans. Amer. Math. Soc. 352 (2000), 855-873. MR 2000c:46023

[7] V. M. Kadets, R. V. Shvidkoy, And D. Werner. Narrow operators and rich subspaces of Banach spaces with the Daugavet property. Studia Math. 147, 269-298 (2001). MR 2002f:46018

[8] K. D. Schmidt. Daugavet's equation and orthomorphisms. Proc. Amer. Math. Soc. 108 (1990), 905-911. MR 90k:47077

[9] R. V. ShvidKoy. Geometric aspects of the Daugavet property. J. Funct. Anal. 176 (2000), 198-212. MR 2001h:46019

[10] D. Werner. Recent progress on the Daugavet property. Irish Math. Soc. Bull. 46 (2001), 77-97. MR 2002i:46014 
[11] P. Wojtaszczyk. Some remarks on the Daugavet equation. Proc. Amer. Math. Soc. 115 (1992), 1047-1052. MR 92k:47041

Faculty of Mechanics and Mathematics, Kharkov National University, Pl. Svobody 4, 61077 Kharkov, UKRAine

E-mail address: vova1kadets@yahoo.com

Current address: Department of Mathematics, Freie Universität Berlin, Arnimallee 2-6, D-14195 Berlin, Germany

E-mail address: kadets@math.fu-berlin.de

Department of Mathematics, Freie Universität Berlin, Arnimallee 2-6, D-14 195 Berlin, Germany

E-mail address: werner@math.fu-berlin.de 